\title{
Machine Vision for Green Citrus Detection in Tree Images
}

\author{
Hiroshi Oкамото and Won Suk LeE* \\ Research Faculty of Agriculture, Hokkaido University, Kita-9, Nishi-9, Kita-ku, Sapporo 060-8589, Japan \\ *Department of Agricultural and Biological Engineering, University of Florida, Rogers Hall, \\ Museum Road, Gainesville, FL 32611, United States
}

(Received February 14, 2010)

\begin{abstract}
The overall goal of this study was to develop a low-cost machine vision system for detecting green fruit on citrus trees. It is difficult to distinguish green citrus fruit from the background leaves using color information because the leaves and fruits are similar in color. Therefore, spatial characteristics such as shape and texture were analyzed. After background extraction, edge detection was performed. Then, circle-template matching was applied to the edge image to detect green fruits. The detection tests revealed that $86 \%$ of the fruit were correctly detected.
\end{abstract}

Keywords : edge detection, image processing, orange, pattern recognition, template matching

\section{INTRODUCTION}

With the help of a GPS receiver, the number of citrus fruits on each tree can be counted and the yield of a citrus grove can be site-specifically estimated. The detection of mature citrus fruit on trees using computer vision has been the focus of several studies. Slaughter and Harrell (1989) developed a classification model that could discriminate oranges from the natural background of an orange grove using only color information in a digital color image for robotic harvesting. Pla et al. (1993) developed a method for characterizing spherical objects in a digital image, and applied it to the detection of citrus fruit under natural conditions. Annamalai and Lee (2003), Annamalai et al. (2004), and Chinchuluun and Lee (2006) developed color vision systems for estimating citrus yield. Regunathan and Lee (2005) differentiated citrus fruit from the background in tree images and estimated the size of the fruit. Chinchuluun and Lee (2006) developed a fruit identification method using the watershed transform to segment and split touching fruit for accurately counting fruit and estimating yield. The target of these studies was mature citrus fruit, which are orange in color and obviously different from leaves. Although it is easy to discriminate orange-colored fruit from green-colored leaves, this type of vision system can only be used just before the harvest season.

Estimating green citrus yield at a much earlier stage could provide growers with multiple benefits, such as adjusting site-specific management practices to increase yield and planning harvest operations in advance to reduce harvesting costs. Early estimation of citrus yield could also affect the future market price. Kane and Lee (2006) determined the optimum wavelengths for discriminating green fruit from leaves by using a spectrophotometer. They subsequently employed a monochromatic near infrared camera equipped with interchangeable optical bandpass filters for identifying green citrus pixels (Kane and Lee, 2007). Okamoto and Lee (2009) detected green fruit

Corresponding author: Hiroshi Okamoto, fax : +81-11-706-2557, e-mail : hiro@bpe.agr.hokudai.ac.jp 


\section{H. OKAMOTO AND W. SUK LEE}

in citrus tree images using hyperspectral imaging with a linear discriminant model and spatial image analysis. However, these studies using special imaging devices such as the near infrared camera and the hyperspectral camera have cost problems.

The overall goal of this study was to develop a low-cost machine vision system for detecting green fruit on citrus trees. We used a red, green, and blue (RGB) color camera, which is cheap because they are widely used not only by professionals but also general users, and their performance is rapidly improving. However, it is difficult to extract green citrus fruit from the background leaves using only color information because the leaves and fruits are similar in color. Therefore, in this study, instead of color information, spatial characteristics such as shape and texture were analyzed for green fruit detection.

Our specific objectives were to:

(a) Extract the foreground portions of green fruit and leaves from the background such as shade, sky, branches and trunks in images of citrus trees;

(b) Find spatial edges in the images to determine the difference between green fruit and leaves without color analysis; and

(c) Detect green citrus fruit in tree images using the template matching method.

\section{MATERIALS AND METHODS}

\section{Image acquisition}

RGB color images of citrus trees were acquired in the experimental orange grove at the University of Florida on August 30, 2006. The target variety of oranges was Hamlin. Images were taken mostly in the shade using a digital still camera (IXY DIGITAL 60, Canon Inc., Tokyo, Japan). The greatest portion of each image was occupied by green fruits and leaves while the remaining portion comprised bright sky, dark shade, branches and trunks.

Manual observation and investigation for developing the algorithm

Prior to developing an algorithm for green citrus detection, we observed and investigated sample images of citrus trees and discovered the following:

(a) It was difficult to distinguish the green fruit from the background leaves using only color information because the leaves and fruits were similar in color;

(b) Color patterns of an image varied according to lighting conditions;

(c) Foreground fruit appeared as a complete circle because citrus fruit is essentially spherical;

(d) Some fruits were occluded by leaves or other fruits. The size of these incomplete fruits was small, and the shape was not a complete circle;

(e) Fruits were larger than leaves; and

(f) The skin of the fruit looked smooth whereas the surface of the leaves was textured with veins.

\section{Algorithm flow for green citrus detection}

Figure 1 shows the flow of green fruit detection. Prior to fruit detection, extraction of the background was performed. After that, edge detection was performed to emphasize the difference between similarly colored fruit and leaves. Finally, circle-template matching was applied to the edge image, and green fruits were detected using fitness of matching. The processing software for this study was developed using Microsoft Visual Studio 2008 with C\# language.

\section{Background removal}

Prior to distinguishing the green fruit from the leaves, the background area was removed (Fig. 1b). The background consisted of bright sky, dark shade, branches and trunks.

In order to remove the areas of bright sky and dark shade, the brightness value of an image pixel in the HSI color space was employed with the thresholding method. The brightness value $I$ was calculated by Eq. (1): 


\section{GREEN CITRUS DETECTION}

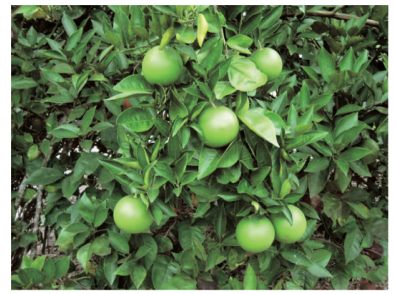

(a) Original image

Background removal

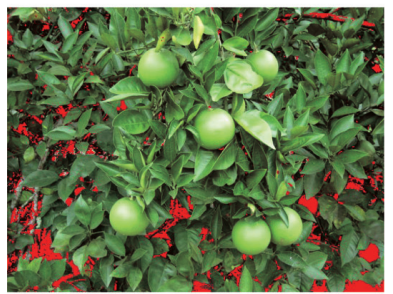

(b) Fruits and leaves

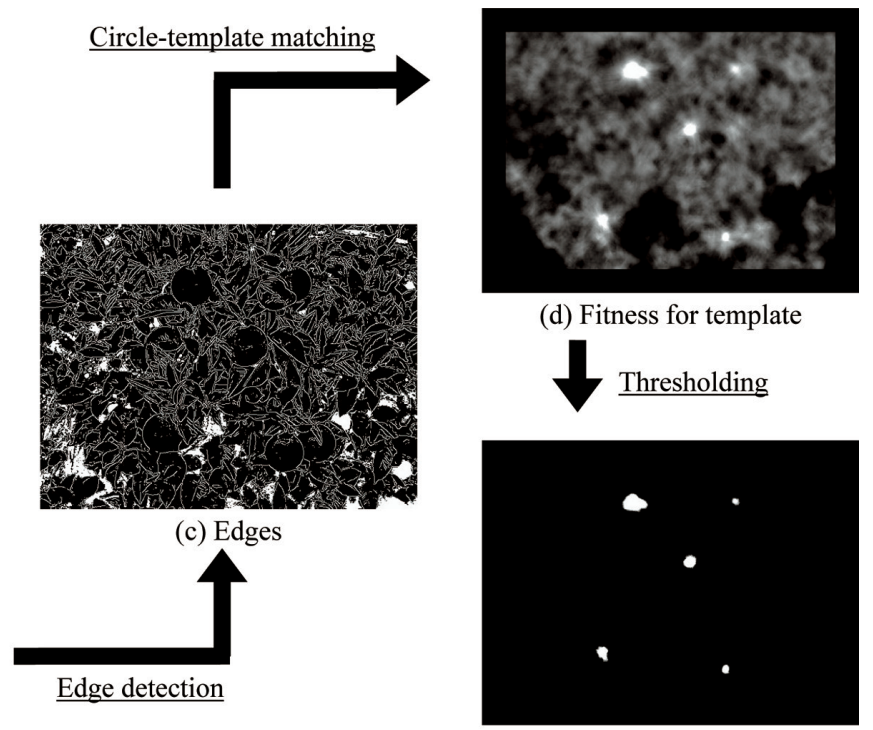

(e) Detected fruits

Fig. 1 Algorithm flow of green citrus detection.

$$
I=\max \{R, G, B\}
$$

where: $R=$ intensity of red channel in RGB color pixels, $G=$ intensity of green channel, $B=$ intensity of blue channel.

$I$ ranges from 0 to 1 theoretically. If $I$ was greater than 0.98 , the pixel was determined as bright sky. If $I$ was less than 0.1 , it was determined as dark shade.

In order to remove the area of branches and trunks, the hue value of a pixel in the HSI color space was employed. Although there are several equations for calculating the hue, in this study, the hue value $H$ was calculated by Eq. (2):

$$
\begin{aligned}
& H=\left\{\begin{array}{l}
60 \cdot \frac{G-B}{I-i}, \text { if } I=R \\
60 \cdot \frac{B-R}{I-i}+120, \text { if } I=G \\
60 \cdot \frac{R-G}{I-i}+240, \text { if } I=B
\end{array}\right. \\
& H=H+360, \text { if } H<0
\end{aligned}
$$

where: $i=\min \{R, G, B\}$

$H$ starts from red at $0^{\circ}$ and ends at red again at $360^{\circ}$. However, in this study, the similarity to pure green color should be measured to distinguish branches and trunks from green fruit and leaves. For that, the original hue value $H$ was converted to a modified value, $H_{g}$ in Eq. (3), to determine the similarity to green.

$$
H_{g}=\left\{\begin{array}{l}
H+60, \text { if } H \leq 120 \\
300-H, \text { if } 120<H<300 \\
H-300, \text { if } H \geq 300
\end{array}\right.
$$

$H_{g}$ ranges from $0^{\circ}$ to $180^{\circ}$, where $180^{\circ}$ is the maximum similarity to pure green. If $H_{g}$ of a pixel was less than $120^{\circ}$, it was determined as a branch or trunk. 


\section{Edge detection}

It is difficult to distinguish green fruit from leaves with color information because of the similarity in color. Therefore, in this study, edge detection was applied for measuring the difference between them. While strong edges were detected on the contour of the fruit, few edges were detected on the skin of the fruit because the skin is smooth. On the other hand, many strong edges were detected on the leaf area because of the presence of many small leaves. In addition, weak edges were also detected as veins inside each leaf. When the edge intensity in an image was displayed using grayscale or black and white, white line segments appeared as edges on the leaf area, and a large spot of blank circles appeared where the fruits were (Fig. 1c). We used a Canny filter for edge detection, which outputs a black and white binary image that shows if a pixel is an edge or not. The threshold for finding initial segments of strong edges was determined to be 100 , and the threshold used for edge linking was also 100.

\section{Circle-template matching}

Since a circle edge with internal blank spot appeared where a fruit was as a result of edge detection, each circle was detected using the template matching method. Figure 2 shows the template that was employed for matching. The edge image was scanned by moving this template, and fitness values between the template and ROI (region of interest) were calculated. The size of the template was scalable by specifying the radius $R$ for adapting to different sizes of fruit. The template consisted of an inside circle portion and an outside ring portion. $R_{1}$ and $R_{2}$, the radiuses of the inside circle and outside ring, are defined in Eq. (4):

$$
\begin{aligned}
& R_{1}=0.85 R \\
& R_{2}=1.15 R
\end{aligned}
$$

The inside circle was used for finding the smooth skin of the fruit, and the outside ring was used for finding the contour edge of the fruit. When the template was located on a fruit, there were few edges in the inside circle due to the smoothness, and many edges in the outside ring due to the fruit contour. On the other hand, when the template was located on a leaf portion, there were many edges of leaves in both the inside circle and the outside ring due to the presence of many small leaves in the ROI.

The index, $F$ in Eq. (5), was defined to calculate template fitness for fruit. $E_{1}$ was divided by $E_{2}$ for normalization because the size of the template was scalable to adapt to the different sizes of fruit.

$$
F=E_{1} / E_{2}
$$

where: $E_{1}=$ the amount of edge intensity in the outside ring, $E_{2}=$ the amount of edge intensity in the inside circle

Values of the fitness $F$ are represented by a grayscale image (Fig. 1d). The portion estimated as fruit is represented by a brighter gray, while the portion estimated as leaves is represented by a

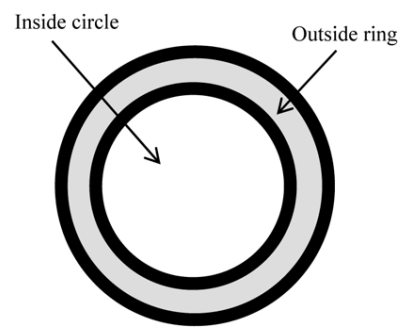

Fig. 2 Circle-template for fruit detection. 


\section{GREEN CITRUS DETECTION}

darker gray. Finally, the location of fruit only was detected by applying the appropriate thresholding method to the fitness image (Fig. 1e).

\section{RESULTS AND DISCUSSION}

Table 1 shows the results of green fruit detection using the sample images. While most of the foreground fruits were correctly detected, some incomplete fruits occluded by leaves or other fruits were incorrectly removed as leaves, whereas a few leaf portions were incorrectly detected as fruit.

Figure 3 shows the case of Image 2. Since Fruits 1, 4, 6 and 7 were at the foreground and the whole fruit appeared, fitness intensity $F$ was strong at their locations and the fruits were correctly detected. Although Fruits 2, 8, 9, 10 and 11 were occluded by leaves or other fruits (notice that just $20 \%$ of Fruit 9 appeared), they were correctly detected. Although Fruits 4 and 5 were overlapping and both of the detected regions were connected, fitness peaks existed at each fruit. Regions $\mathrm{A}$ and $\mathrm{B}$ on the leaves were incorrectly detected as fruit because the leaf edges were not detected and large blank spots appeared there. Fruit 3 was incorrectly removed as a leaf because it was occluded by leaves and another fruit and showed no more than $15 \%$ of itself, provided that its shape was not a complete circle.

Figure 4 shows the case of Image 1. Complete Fruits 1, 3, 4 and 5 were correctly detected. Although Fruit 2 was half occluded by leaves, it was correctly detected. Leaf A was correctly re-

Table 1 Results of green fruit detection tests.

\begin{tabular}{ccccc}
\hline Image No. & $\begin{array}{c}\text { Radius of template } \\
\text { (pixels) }\end{array}$ & $\begin{array}{c}\text { Number of complete } \\
\text { fruit (Detected/ } \\
\text { Actual) }\end{array}$ & $\begin{array}{c}\text { Number of incomplete } \\
\text { fruit occluded by } \\
\text { leaves and fruit } \\
\text { (Detected / Actual) }\end{array}$ & $\begin{array}{c}\text { Number of leaf } \\
\text { regions incorrectly } \\
\text { detected as fruit }\end{array}$ \\
\hline 1 & 35 & $4 / 4$ & $1 / 2$ & 0 \\
2 & 35 & $4 / 4$ & $6 / 7$ & 3 \\
3 & 35 & $6 / 6$ & $6 / 7$ & 0 \\
4 & 45 & $3 / 5$ & $0 / 0$ & 0 \\
5 & 55 & $3 / 3$ & $3 / 4$ & 0 \\
Overall & $20 / 22$ & $16 / 20$ & 3 \\
\hline
\end{tabular}

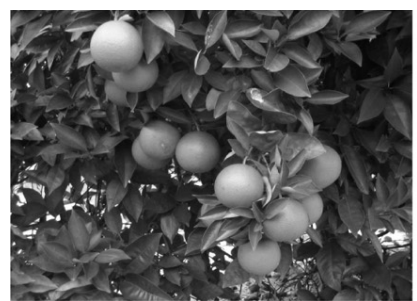

(a) Original image

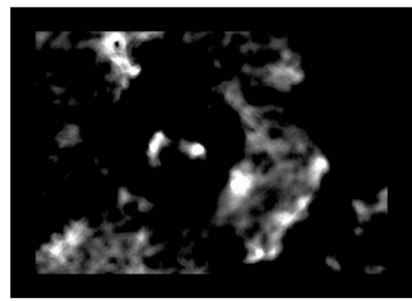

(c) Fitness for template

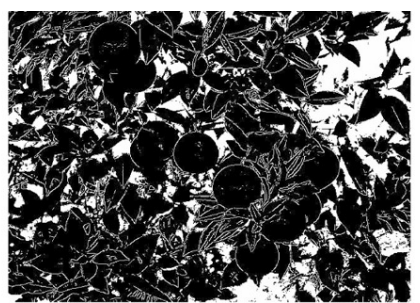

(b) Edges

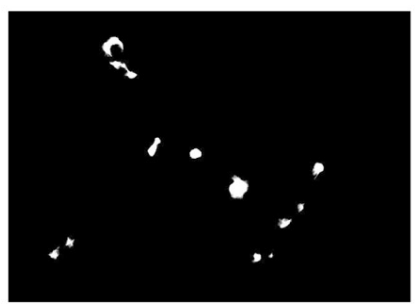

(d) Detected fruits

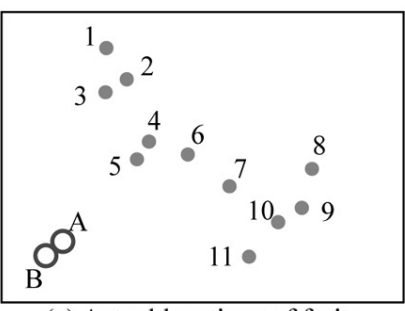

(e) Actual locations of fruits

Fig. 3 Case of Image 2. 


\section{H. OKAMOTO AND W. SUK LEE}

moved even though its shape was circular like a fruit, because its leaf veins appeared as edges. Fruit 6 was incorrectly removed as a leaf because the edges of the leaf occluding it influenced the fruit detection.

Figure 5 shows the case of Image 4. Fruits 2, 3 and 4 were correctly detected. Although Fruit 1 was at the foreground and the shape of its contour was a complete circle, it was incorrectly removed as a leaf because of many noisy edges on its skin due to slight unevenness. In order to resolve the problem, noise reduction was applied. Fruit 5 was incorrectly removed as a leaf because it was smaller than the template.

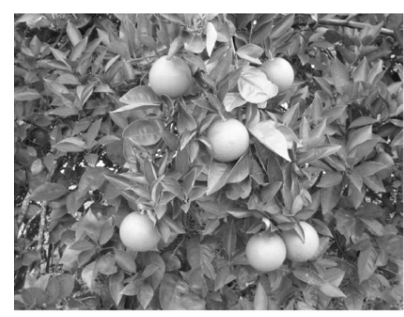

(a) Original image

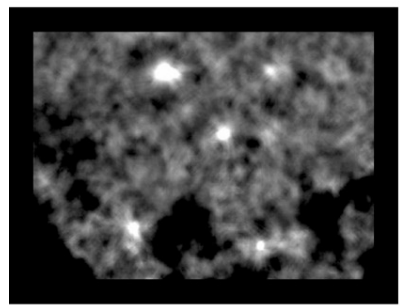

(c) Fitness for template

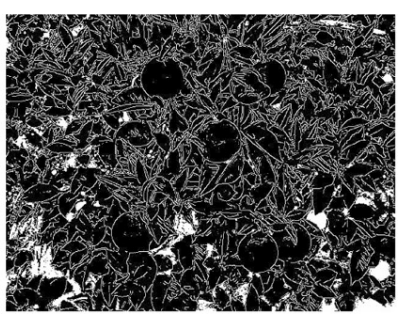

(b) Edges

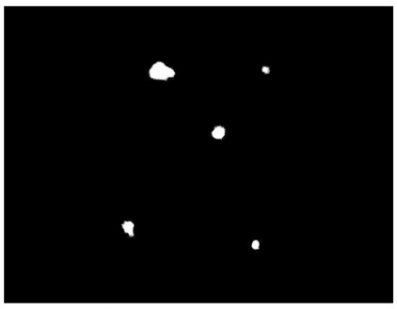

(d) Detected fruits

Fig. 4 Case of Image 1.

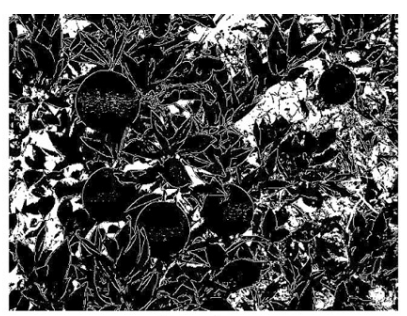

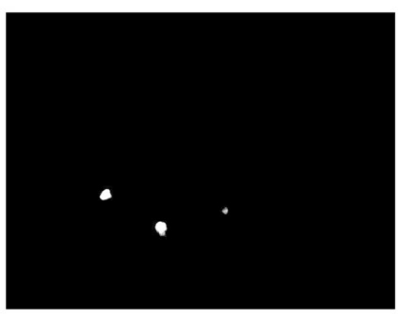

(d) Detected fruits (b) Edges

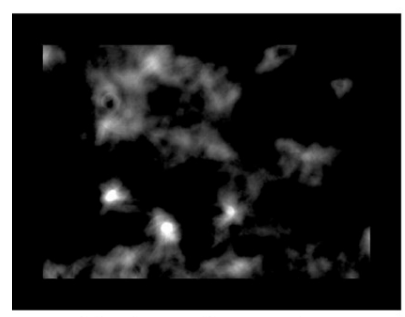

(c) Fitness for template

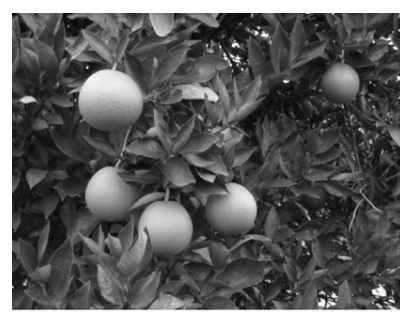

(a) Original image

Fig. 5 Case of Image 4.

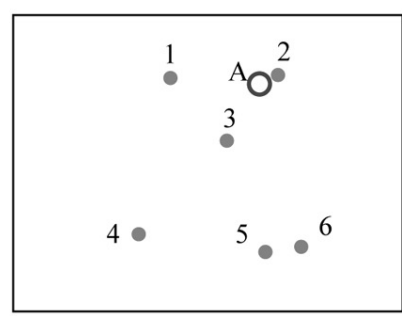

(e) Actual locations of fruits 


\section{GREEN CITRUS DETECTION}

\section{CONCLUSIONS}

The overall goal of this study was to develop a machine vision system for detecting green fruit on citrus trees. For a low-cost system, the study employed an RGB color camera. It is difficult to extract green citrus fruit from background leaves using only color information because the leaves and fruits are similar in color. Therefore, in this study, instead of color information, spatial characteristics such as shape and texture were analyzed for green fruit detection.

Prior to fruit detection, extraction of the background was performed. After that, Canny-filter edge detection was performed to determine the difference between similarly colored fruit and leaves. Finally, circle-template matching was applied to the edge image, and green fruits were detected with fitness of matching.

The detection tests revealed that $91 \%$ of the complete fruit and $80 \%$ of the incomplete fruit occluded by others were correctly detected.

We thank Dr. F. Davies in the Horticultural Science Department and Mr. John Thomas, Grove Manager, for permitting the use of the University of Florida Citrus Grove. We also thank Mr. Kevin E. Kane, a graduate research assistant at the University of Florida, for his assistance.

\section{REFERENCES}

Annamalai, P., Lee, W. S. 2003. Citrus yield mapping system using machine vision. ASAE Paper No. 031002. ASAE, St. Joseph, MI, USA.

Annamalai, P., Lee, W. S., Burks, T. F. 2004. Color vision system for estimating citrus yield in real-time. ASAE Paper No. 043054. ASAE, St. Joseph, MI, USA.

Chinchuluun, R., Lee, W. S. 2006. Citrus yield mapping system in natural outdoor scenes using the watershed transform. ASABE Paper No. 063010. ASABE St. Joseph, MI, USA.

Kane, K. E., Lee, W. S. 2006. Spectral sensing of different citrus varieties for precision agriculture. ASABE Paper No. 061065. ASABE, St. Joseph, MI, USA.

Kane, K. E., Lee, W. S. 2007. Multispectral imaging for in-field green citrus identification. ASABE Paper No. 073025. ASABE, St. Joseph, MI, USA.

Okamoto, H., Lee, W. S. 2009. Green citrus detection using hyperspectral imaging. Comput. Electron. Agric. 66: $201-208$.

Pla, F., Juste, F., Ferri, F. 1993. Feature extraction of spherical objects in image analysis: an application to robotic citrus harvesting. Comput. Electron. Agric. 8: 57-72.

Regunathan, M., Lee, W. S. 2005. Citrus yield mapping and size determination using machine vision and ultrasonic sensors. ASAE Paper No. 053017. ASAE, St. Joseph, MI, USA.

Slaughter, D. C., Harrell, R. C. 1989. Discriminating fruit for robotic harvest using color in natural outdoor scenes. Trans. ASAE 32: 757-763. 